\title{
Validation of serum creatinine-based formulae in pediatric renal transplant recipients
}

\author{
Omar Alkandari ${ }^{1}$, Diane Hebert ${ }^{1}$, Valerie Langlois ${ }^{1}$, Lisa A. Robinson ${ }^{1}$ and Rulan S. Parekh ${ }^{1}$
}

BACKGROUND: Accurate monitoring of kidney function is important post-renal transplant; however, the routine use of measured glomerular filtration rate (GFR) or addition of newer serum markers is prohibitively expensive for routine clinical use, especially in children. We validated the modified Schwartz formula in pediatric renal transplant recipients across a range of demographic and clinical characteristics.

METHODS: In a retrospective cohort study with nested cross-sectional analysis, we compared 505 measurements of estimated GFR using serum creatinine to simultaneous diethylenetriaminepentaacetic acid (DTPA) nuclear GFR (nGFR) measurements from 173 pediatric kidney transplant recipients who were $<18$ years of age from 1 January 2001 to 31 December 2012 accounting for repeated measures.

RESULTS: Among 173 children, 62\% were males, 85\% with nGFR of $\geq 60 \mathrm{ml} / \mathrm{min} / 1.73 \mathrm{~m}^{2}$, and the median age at transplant was 13.6 years (interquartile range 8.3-16 years). Overall, the modified Schwartz and Pottel formulae had better bias $\left(0.07\right.$ and $-0.03 \mathrm{ml} / \mathrm{min} / 1.73 \mathrm{~m}^{2}$, respectively) and accuracy within 30\% (both 84.4\%) in comparison to Lyon and Zappitelli formulae. The 30\% accuracy varied for girls and children $<5$ and $>15$ years.

CONCLUSION: Modified Schwartz is a practical, non-invasive, and a valid bedside tool that provides a valid measurement of GFR in pediatric kidney transplant recipients.

K idney function is best assessed and monitored by determining the glomerular filtration rate (GFR). Changes in GFR affect drug dosing and increase the surveillance for progressive kidney disease and systemic issues, such as anemia and bone disease after kidney transplantation. Accurate measurement and close monitoring of GFR are important in kidney transplant recipients. GFR is acknowledged as the primary indicator of renal graft function (1), it is an independent risk factor for cardiovascular mortality, which is a main cause of death in both pediatric and adult kidney transplant recipients $(2,3)$, and an indicator of long-term graft survival (4). In addition, therapeutic agents for the treatment of graft rejection use GFR as an outcome measure to determine efficacy.

Inulin clearance, the gold standard for measuring GFR, is impractical in the clinical setting. Nuclear GFR (nGFR) has been validated and widely used as the accepted standard of measured GFR (5), however, it is costly, time consuming, and requires an intravenous or subcutaneous infusion. An additional concern is the repeated exposure to radiation, which, in the pediatric population carries potential latent risks including malignancy (6). There are also novel markers of kidney function such as cystatin $\mathrm{C}$, but the additional cost for frequent testing precludes routine clinical use in most countries (7). Due to these limitations, several serum creatinine (SCr)-based formulae for estimated GFR (eGFR) have been developed and recommended by the Kidney Disease Outcomes Quality Initiative (K/DOQI) guidelines (1). The 2009 Kidney Disease: Improving Global Outcomes (KDIGO) clinical practice guideline for the care of kidney transplant recipients suggests use of an eGFR whenever $\mathrm{SCr}$ is measured using the Schwartz formula for children and adolescents based on level 2C grade of evidence. However, there is limited empirical evidence to support this guideline (8).

In children, the Schwartz formula has been used since 1976 despite the lack of precision (9). Thus, the new modified Schwartz formula (mod-Schwartz), updated in 2009, demonstrates improved precision compared to iohexol GFR and can be used in children with mild to moderate chronic kidney disease (CKD) with a GFR between 30 and $90 \mathrm{ml} / \mathrm{min} / 1.73 \mathrm{~m}^{2}$ (9-11). Two recent large analyses (738 GFRs from 503 children with primarily non-CKD; 1,074 GFRs from 469 CKD children including 111 renal transplant recipients) demonstrate good agreement of mod-Schwartz to iothalamate nGFR and inulin clearance, respectively $(12,13)$. In addition, a comparison to iothalamate clearance with cystatin $\mathrm{C}$ or creatinine eGFR-based measures among various ethnic groups demonstrated modest-to-good correlation overall (14-16). Although these studies strengthen the clinical utility of using the eGFR equation for both CKD and non-CKD patients, they did not address a large number of transplant recipients. De Souza and colleagues have recently evaluated 
and compared the performance of SCr-based formulae (modSchwartz and Schwartz-Lyon) along with cystatin C-based (Hoek and Filler) and combined SCr-cystatin C-based formulae (CKiD 2012 and Zappitelli) in pediatric renal transplant recipients, however, they did not tackle the possible variation by age, gender, and clinical characteristics, which all may influence GFR (17). In addition, two other heightindependent formulae, Zappitelli and Pottel, were recently validated $(18,19)$.

There is emerging evidence demonstrating that estimating GFR using serum cystatin C-based equations correlate better than SCr-based formulae in adults and children (20-25). Studies to date indicate a modest agreement of the modSchwartz and measured GFR $(10,12,13,17,26)$, and measurement of serum cystatin $\mathrm{C}$ is not widely available, limiting the routine clinical use; thus, our goal was to confirm that the mod-Schwartz formula is a valid bedside SCr-based formula to estimate GFR in larger populations of kidney transplant recipients and across a range of demographic and clinical characteristics.

\section{METHODS}

\section{Study Population and Design}

This was retrospective cohort study with nested cross-sectional analysis at the time of GFR measurements. The study included all kidney transplant recipients below the age of 18 years with available data on nGFR after transplantation at the Hospital for Sick Children in Toronto, Canada between 1 January 2001 and 31 December 2012. Multiorgan transplant recipients were excluded. The study protocol was approved by the Research Ethics Board at the Hospital for Sick Children. The requirement for participant consent was waived.

\section{Clinical Data}

SCr and children's height measurements were performed on the same day of nGFR measurement. Data were collected from the electronic patient record during outpatient clinic visits and include demographic and clinical characteristics. Laboratory data at the time of the nGFR measurement were collected from the participants' electronic records.

\section{SCr and eGFR measures}

SCr (micromol/l $(\mu \mathrm{M}))$ was measured by a dry slide enzymatic creatinine assay on Ortho VITROSR 5.1 FS Chemistry System (Ortho Clinical Diagnostics, Markham, Ontario, Canada). The modSchwartz uses the new standardized enzymatic creatinine assay method (10). As of 17 March 2008, the creatinine assay was calibrated to be traceable to an isotope dilution mass spectrometry (IDMS) reference method, a critical step toward creatinine standardization. This calibration yielded lower SCr levels by $15 \%$ compared to previous measurements in an internal hospital laboratory validation study performed by the Department of Pathology and Laboratory Medicine at The Hospital for Sick Children (data not shown). To account for this variation, a correction factor of 0.85 was used for all SCr values obtained before 17 March 2008 similar to other reported calibration methods (1). Stratified analyses conducted to test for differences before and after 2008 did not find any significant differences in the results (data not shown).

Estimated GFR using two height-dependent (mod-Schwartz and Schwartz-Lyon (Lyon) and two height-independent (Zappitelli and Pottel) SCr-based formulae was studied in children including: (a) mod-Schwartz $(36.5 \times \mathrm{Ht}$ in $\mathrm{cm} / \mathrm{SCr}$ in $\mu \mathrm{M})(10,16)$ (b) Lyon formula (13) $(36.5 \times \mathrm{Ht}$ in $\mathrm{cm} / \mathrm{SCr}$ in $\mu \mathrm{M})$ for boys $\geq 13$ years and $(32.5 \times \mathrm{Ht}$ in $\mathrm{cm} / \mathrm{SCr}$ in $\mu \mathrm{M})$ for boys $<13$ years and in all girls, (c) Zappitelli et al. (18) (inverse $\ln$ of: $8.067+(1.034 \times \ln [1 / \mathrm{SCr}$ $(\mu \mathrm{M})])+(0.305 \times \ln$ [age (years)] $)+0.064$ if male) and (d) Pottel et al. (19) $(107.3 /(\mathrm{SCr} / Q))$ where $Q$ is the median SCr concentration for children based on age and sex (see reference for different $Q$ values) (27).

\section{Nuclear GFR}

As per hospital protocol, nGFR $\left(\mathrm{ml} / \mathrm{min} / 1.73 \mathrm{~m}^{2}\right)$ was measured by a two-point Technetium-99m-diethylene-triamine-pentaacetate $\left({ }^{99 m}\right.$ Tc DTPA) with a dose of 37 millibecquerel $(\mathrm{mBq}) / 1$ millicurie $(\mathrm{mCi})$ to $111 \mathrm{mBq} / 3 \mathrm{mCi}$. The first blood sample was taken $90 \mathrm{~min}$ after the intravenous tracer injection and a second sample at least $60 \mathrm{~min}$ later. Blood samples were drawn peripherally from a different site than the injection site. Per protocol, baseline nGFR was typically performed within the first few months post transplant and annually thereafter.

\section{Analysis}

Descriptive statistics of the entire study population were expressed as the median and interquartile range (IQR) for continuous variables and proportions for categorical variables. To attain normality for subsequent analyses, nGFR and eGFR were log-transformed. Repeated GFR measures from the same subjects may be correlated (i.e., each measure was not independent from the other), thus, we have conducted the analyses in two ways to account for repeated studies within a single child: (i) clustering by person as recommended by Bland et al. $(28,29)$ (main analyses) and (ii) a sensitivity analyses conducted among randomly selected 173 studies (one study per child). We report on the mean bias (mean difference between log-nGFR and log-eGFR), precision (standard deviation (SD) of bias), and accuracy within 10 or $30 \%$ deviation from nGFR (percentage of estimates that differed from the measured GFR by more than $10 \%$ and $30 \%$, respectively) among the entire population and by subgroups. These criteria were based on the K/DOQI CKD guidelines and CKD-EPI consortium publications with uniform criteria in reporting differences in eGFR measurements compared to nGFR $(23,30)$. Bland-Altman analyses were conducted to determine the agreement between the average difference of log-nGFR minus log-eGFR and the average of log-nGFR and log-eGFR ((log-nGFR $+\log$-eGFR)/2)) with corresponding lines 2 SD above and below zero (31). Paired Student's $t$-test was used to assess whether the bias and accuracy (10 and $30 \%$ ) of Lyon, Zappitelli, and/or Pottel formulae were significantly different $(P$-value $<0.05)$ from those of modSchwartz formula. The STATA statistical software package (STATA 12, STATA Corporation, College Station, TX) was used for all analyses.

\section{RESULTS \\ Study Population}

Clinical characteristics and baseline transplant information are presented in Table 1. Between 1 January 2001 and 31 December 2012, 173 incident pediatric kidney transplant recipients had 505 nGFR measurements with a corresponding SCr on the same day. The majority of participants (69\%) were older than 10 years at the time of transplant. The study population consisted predominantly of males (62\%), those with congenital anomalies of the kidney and urinary tract (46\%), first kidney transplant recipients (98\%), and deceased donors grafts (54\%). Overall, the median nGFR was higher than eGFR using mod-Schwartz formula at baseline. Eightythree percent of participants had the nGFR $\geq 60 \mathrm{ml} / \mathrm{min} /$ $1.73 \mathrm{~m}^{2}$. Participants had a median of 2.5 nGFRs (range of 1-11) performed per participant until transferred at age 18 


\section{Articles | Alkandari et al.}

Table 1. Baseline demographics and clinical characteristics of 173 pediatric kidney transplant recipients (2001-2012)

\begin{tabular}{lcc}
\hline & $n(\%)$ & Median (IQR) \\
\hline All patients & $173(100)$ & $13.2(8.1-15.7)$ \\
Age at transplant (years) & $23(13.3)$ & $35(20.2)$ \\
$<5$ & $52(30.1)$ & $63(36.4)$ \\
$5-9.9$ & $108(62)$ \\
$10-14.9$ & \\
$>15$ & $168(97.1)$ \\
Male & $4(2.3)$ \\
Kidney transplant & $1(0.6)$ \\
First allograft & \\
Second allograft & \\
Third allograft & $80(46)$ \\
Etiology of ESRD & $45(26)$ \\
CAKUT & $22(13)$ \\
Glomerular disorders & $26(15)$ \\
Genetic disorders &
\end{tabular}

Donor type

$\begin{array}{ll} & 79(46) \\ \text { Living } & 94(54)\end{array}$

Year of transplant

$\begin{array}{ll}2001-2004 & 58(33.5) \\ 2005-2008 & 56(32.4) \\ 2009-2012 & 59(34.1)\end{array}$

$n G F R\left(\mathrm{ml} / \mathrm{min} / 1.73 \mathrm{~m}^{2}\right)$

$\geq 90$

84 (67-104)

$\geq 60$ and $<90$

$\geq 30$ and $<60$

$\geq 15$ and $<30$

$<15$

$e G F R^{\mathrm{a}, \mathrm{b}}\left(\mathrm{ml} / \mathrm{min} / 1.73 \mathrm{~m}^{2}\right)$

$\geq 90$

79 (63-94.7)

$\geq 60$ and $<90$

$\geq 30$ and $<60$

$\geq 15$ and $<30$

$<15$

147 (126-158)

Height $^{\mathrm{a}}(\mathrm{cm})$

Weight ${ }^{\mathrm{a}}(\mathrm{kg})$

BMI $z$-score ${ }^{a}$

Creatinine $e^{\mathrm{a}}(\mu \mathrm{M})$

CAKUT, congenital anomalies of the kidney and urinary tract; eGFR, estimated glomerular filtration rate; IQR, interquartile range; $\mathrm{nGFR}$, nuclear glomerular filtration rate.

At time of nGFR measurement $(n=173)$.

beGFR using mod-Schwartz formula. years. The median time from transplant to the first nGFR was 34 days (IQR 16-56 days).

\section{Performance of Modified Schwartz Formula}

Table 2 displays the bias $\left(\mathrm{ml} / \mathrm{min} / 1.73 \mathrm{~m}^{2}\right)$, SD of bias $(\mathrm{ml} /$ $\min / 1.73 \mathrm{~m}^{2}$ ), and accuracy (30 and $10 \%$ ) using the modSchwartz formula. In the overall cohort, the mod-Schwartz formula showed the bias of $0.07 \pm 0.21$, the $30 \%$ accuracy value of 84.4 and $10 \%$ accuracy value of 36.4 . Bland-Altman plots (Figure 1a) confirmed good agreement with measured and estimated GFR.

There was a higher bias in children older than 15 years, male, and those with CKD stage-I (nGFR $\geq 90 \mathrm{ml} / \mathrm{min} /$ $1.73 \mathrm{~m}^{2}$ ). The highest $30 \%$ accuracy values were among the children between the ages of 10 and 15 years, those with CKD stage-II (nGFR 60-89 $\mathrm{ml} / \mathrm{min} / 1.73 \mathrm{~m}^{2}$ ), extreme BMI (BMI $z$ score less than -2 and greater than +2 ), genetic causes of ESRD, and the measurements 3 months after transplantation.

Sensitivity analyses were conducted to account for a single measure per child that demonstrates similar results (Supplementary Table S1 online, Supplementary Table S2 online, and Supplementary Figure S1 online).

\section{Performance of Lyon, Zappitelli, and Pottel Formulae}

Table 3 displays comparison between mod-Schwartz and Lyon, Zappitelli, and Pottel formulae with all repeated measures and only comparing one measure per child. When comparing these three formulae to mod-Schwartz formula, the biases and 30\% accuracy differed significantly from modSchwartz formula. When compared to mod-Schwartz, the results revealed underestimation with Pottel and Zappitelli formulae $(-0.03$ and -0.1 , respectively), whereas modSchwartz, Lyon, and Pottel were more accurate in estimating GFR showing $30 \%$ accuracy of 84.4, 84.4, and $85 \%$ respectively compared to Zappitelli. Overall, the BlandAltman plots (Figure 1b,c) confirmed good agreement of these formulae with measured and estimated GFR.

\section{DISCUSSION}

Repeated monitoring of GFR is critical in the clinical management of children after renal transplantation. The mod-Schwartz equation of eGFR using creatinine in children has been validated in CKD and renal transplant recipients, and now those results extend to a wider range of GFR, across demographic and clinical characteristics $(10,13)$. Using a valid formula to estimate GFR in pediatric renal transplant recipients will allow assessment of GFR without exposing children to the radiation exposure, complications, and inconveniences associated with measured GFR.

Overall, there is a good agreement between measured nGFR using DTPA and estimating GFR using mod-Schwartz formula across the spectrum of GFR. This concurs with prior studies, however, in our analysis mod-Schwartz's 30\% accuracy was slightly higher than reported $(13,17)$. In our study, the $30 \%$ accuracy for the mod-Schwartz formula varied for females and for children less than 5 years and older than 
Table 2. Bias, precision, and accuracy ( $30 \%$ and $10 \%$ ) of eGFR using modified Schwartz formula of 505 nGFR measurements among 173 children accounting for repeated measures

\begin{tabular}{lccccc}
\hline & $n(\%)$ & Bias $^{\mathrm{a}}$ & $\begin{array}{c}\text { SD of } \\
\text { bias }^{\mathrm{a}}\end{array}$ & $\begin{array}{c}30 \% \\
\text { Accuracy }\end{array}$ & $\begin{array}{c}10 \% \\
\text { Accuracy }\end{array}$ \\
\hline All GFR & $173(100)$ & 0.07 & 0.21 & 84.4 & 36.4
\end{tabular}

Age at $n G F R$ (years)

$\begin{array}{llrrrr}<5 & 12(7) & -0.1 & 0.15 & 83.3 & 25.0 \\ 5-10 & 30(17.3) & 0.05 & 0.19 & 86.7 & 46.7 \\ 10-15 & 54(31.2) & 0.05 & 0.19 & 92.6 & 35.2 \\ >15 & 77(44.5) & 0.12 & 0.22 & 77.9 & 35.6\end{array}$

Sex

$\begin{array}{lrllll}\text { Male } & 108(62) & 0.09 & 0.21 & 83.3 & 37.0 \\ \text { Female } & 65(38) & 0.05 & 0.2 & 86.2 & 35.4\end{array}$

$\begin{array}{llllll}\text { CKD stages }^{\mathrm{b}} & & & & & \\ \text { Stage I } & 72(41.6) & 0.13 & 0.22 & 79.1 & 30.5 \\ \text { Stage II } & 75(43.4) & 0.05 & 0.18 & 89.3 & 44.0 \\ \text { Stages III-V } & 26(15) & -0.05 & 0.2 & 84.6 & 30.8\end{array}$

BMI Z-score

$\begin{array}{lcclcl}>2 & 15(8.7) & 0.05 & 0.19 & 93.3 & 33.3 \\ -2 \text { to } 2 & 150(86.7) & 0.08 & 0.22 & 82.7 & 36 \\ <-2 & 8(4.6) & 0.04 & 0.1 & 100 & 50\end{array}$

\section{Diagnosis}

$\begin{array}{llllll}\text { CAKUT } & 80(46) & 0.1 & 0.22 & 80 & 33.8 \\ \text { Glomerular } & 45(26) & 0.03 & 0.19 & 88.9 & 42.2 \\ \text { Genetics } & 22(13) & 0.07 & 0.22 & 90.9 & 36.4 \\ \text { Others } & 26(15) & 0.07 & 0.2 & 84.6 & 34.6\end{array}$

nGFR post transplant

\begin{tabular}{lrrrrr}
$\begin{array}{l}\text { Initial } \\
3 \text { months }\end{array}$ & $58(33.5)$ & 0.05 & 0.26 & 77.6 & 22.4 \\
$\begin{array}{l}\text { After } \\
3 \text { months }\end{array}$ & $115(66.5)$ & 0.08 & 0.18 & 87.8 & 43.5 \\
\hline
\end{tabular}

CAKUT, congenital anomalies of the kidney and urinary tract; eGFR, estimated GFR; nGFR, nuclear GFR.

a eGFR and nGFR were log-transformed $\left(\mathrm{ml} / \mathrm{min} / 1.73 \mathrm{~m}^{2}\right)$.

${ }^{b}$ Based on nGFR measurement (stage I: $\geq 90$, stage II: 60-89, stages III-V: $<60 \mathrm{ml}$ / $\min / 1.73 \mathrm{~m}^{2}$ ).

15 years, which is important to understand the variability of eGFR when using the mod-Schwartz clinically. These disparities could be attributed to the fact that boys and older children have more muscle mass than girls and small children $(32,33)$, and thus SCr-based formula will underestimate true GFR in males and older children. We could not account for pubertal differences that may also impact eGFR measurements (33). These biases are also evident with the original
Schwartz formula, where different coefficients are used in the formula to account for the changes in muscle mass for infants and adolescent boys (34). In an attempt to account for this variability with muscle mass, the Lyon formula uses the $k$ coefficient of the mod-Schwartz of 32.5 for girls and boys $<13$ years, and also 36.5 for boys $\geq 13$ years (13). The addition of the coefficient further improves the mod-Schwartz performance in CKD and renal transplant recipients $(13,17)$; however, we could not reproduce these findings that reflects the noise in estimating GFR with age-based equations.

Children with GFR between 60 and $89 \mathrm{ml} / \mathrm{min} / 1.73 \mathrm{~m}^{2}$ have the best $30 \%$ accuracy, whereas patients with CKD stage I (GFR $\geq 90 \mathrm{ml} / \mathrm{min} / 1.73 \mathrm{~m}^{2}$ ) have the worse bias in comparison to other GFR categories (CKD stage II and stages III-V). This likely reflects the population in whom the formula was developed. De Souza et al. have found similar pattern using the Lyon-derived eGFR but not with the mod-Schwartz, but in a very limited sample of nine children. Also, it is not known how accurate the formulae are when GFR exceeds $120 \mathrm{ml} /$ $\min / 1.73 \mathrm{~m}^{2}$. Our data differ from other studies of CKD, renal transplant, and cancer patients where those with a GFR $>90 \mathrm{ml} / \mathrm{min} / 1.73 \mathrm{~m}^{2}$ had the best accuracy $(12,13)$.

Comparisons of children with obstructive uropathy who have presumed high-output kidney failure to glomerular disorders with mostly oligo-anuria demonstrate a similar 30\% accuracy of mod-Schwartz. Despite the lack of residual kidney function information, the results indicate the consistency of the mod-Schwartz regardless of the underlying renal pathology. Importantly, the mod-Schwartz eGFR in the first 3 months post transplant is less accurate than eGFR after the first 3 months, possibly due to many early mild acute tubular necrosis, fluctuations in fluid status, and medications that affect SCr, such as cotrimoxazole or unstable calcineurin inhibitor levels. Understanding the limitations of the estimating equation in specific clinical situations remains important for clinical management.

A recent paper on the KIMONO study studied different eGFR formulae (SCr, cystatin C, and SCr-cystatin C-based formulae) in comparison to inulin clearance in children with single kidneys and found that the mean bias for eGFR using mod-Schwartz formula is $0.4 \mathrm{ml} / \mathrm{min} / 1.73 \mathrm{~m}^{2}$ with $90 \%$ and $33 \%$ of values within $30 \%$ and $10 \%$ of inulin GFR, respectively (26). Children with a single kidney after transplantation typically have native kidneys remaining that results in higher bias and less accuracy. The differences in our study cohort and the reported studies reflect the heterogeneity of the study populations, the improved precision using cystatin $\mathrm{C}$, and also potential impact of residual kidney function. Although cystatin C-based formulae correlate better to measured GFR than SCr-based formulae in native kidneys, Papez et al. demonstrated similar GFR correlation between mod-Schwartz and cystatin C-based formulae in 78 pediatric renal transplant recipients (15). Future studies are needed to clarify the use of cystatin C-based equations in a large cohort of pediatric transplant recipients, given the frequency of screening and additional cost. 
a Bland-Altman plot of mod-Schwartz GFR \& nGFR

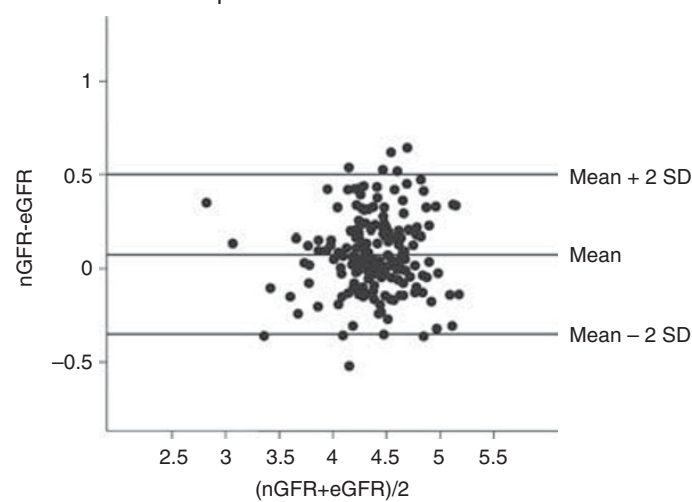

C Bland-Altman plot of Zappitelli GFR \& nGFR

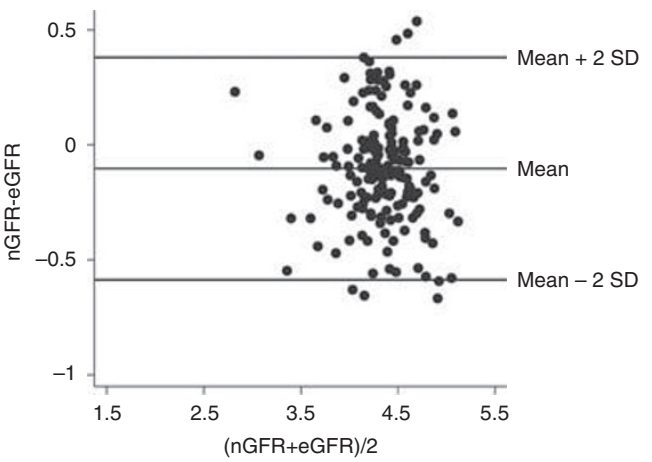

b Bland-Altman plot of Lyon GFR \& nGFR

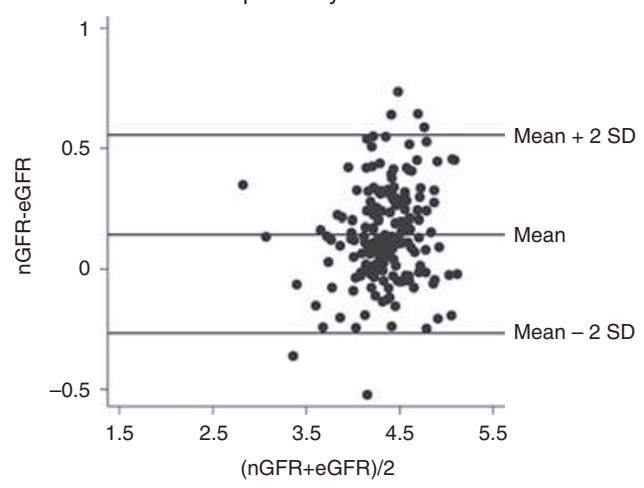

d Bland-Altman plot of Pottel GFR \& nGFR

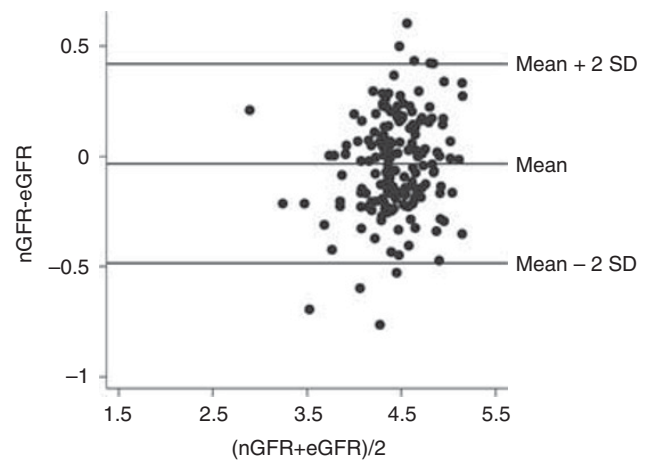

Figure 1. Bland-Altman plots of nGFR and serum creatinine-based eGFR of 505 nGFR measurements among 173 children accounting for repeated measures using (a) modified-Schwartz formula, (b) Lyon formula, (c) Zappitelli formula, and (d) Pottel formula. Mean bias and the standard deviation (SD) bias are represented by three horizontal solid lines. The theoretical 100\% agreement between two methods lie between these two SD lines. Estimated GFR and nGFR were log-transformed.

Table 3. Bias, precision, and accuracy (30\% and 10\%) of mod-Schwartz, Lyon, Zappitelli, and Pottel formulae of 505 nGFR measurements among 173 children accounting for repeated measures

\begin{tabular}{lcccc}
\hline & \multicolumn{4}{c}{ SCr-based formulae } \\
\cline { 2 - 5 } Variable & Mod-Schwartz & Lyon & Zappitelli & \multicolumn{1}{c}{ Pottel } \\
\hline Bias $^{\mathrm{a}}$ & 0.07 & $0.14(0.002)^{\mathrm{b}}$ & $-0.1(<0.001)^{\mathrm{b}}$ & 0.24 \\
SD of bias & 0.21 & 0.21 & $72.8(0.002)^{\mathrm{b}}$ & 0.22 \\
$30 \%$ Accuracy & 84.4 & $85(<0.001)^{\mathrm{b}}$ & $29.5(0.2)^{\mathrm{b}}$ & $84.4(<0.001)^{\mathrm{b}}$ \\
$10 \%$ Accuracy & 36.4 & $33.5(<0.001)^{\mathrm{b}}$ & $31.2(0.07)^{\mathrm{b}}$ \\
\hline
\end{tabular}

eGFR, estimated GFR; nGFR, nuclear GFR; SCr, serum creatinine.

aeGFR and nGFR were log transformed $\left(\mathrm{ml} / \mathrm{min} / 1.73 \mathrm{~m}^{2}\right)$.

${ }^{\mathrm{b}}$ P-values for bias and accuracy comparisons between Lyon, Zappitelli, and Pottel vs. Mod-Schwartz using "paired Student's t-test" or Chi-square-test are given in parentheses.

Despite the validation of mod-Schwartz formula in renal transplant recipients, measured GFR still remains the best standard and should be considered in situations where modSchwartz may not be accurate such as in children with lower muscle mass, those using potent nephrotoxic medications and conditions when acute kidney function deterioration occurs. Several limitations are present in our study. The study cohort had very few children with advanced CKD, in which the modSchwartz formula is developed and thus the range where the precision is better (10). Another limitation is the absence of late, third sampling point of DTPA that can explain the overestimation of GFR especially in those with very low GFR, which was not the case in our study population. We also could not test the use of serum cystatin-C in this historical cohort, which may further improve precision of eGFR and needs to be tested prospectively (35). In addition, we could not account for drug effects on creatinine metabolism or GFR including CNIs or cotrimoxazole; however, all patients receive 
tacrolimus and a standard course of cotrimoxazole prophylaxis. The main advantage of our study is that our cohort is large with many repeated measures over a long follow-up and can be generalizable to other pediatric transplant populations. Also, SCr is available almost everywhere in the world, so validating the simple and widely used mod-Schwartz is important for pediatric nephrologists following renal transplant recipients.

In conclusion, modified Schwartz is a valid bedside equation to assess GFR in pediatric renal transplant recipients. Good performance characteristics of the modSchwartz eGFR confirms clinical utility in both transplant and non-transplant CKD patients, with the exception of CKD stage I $\left(>90 \mathrm{ml} / \mathrm{min} / 1.73 \mathrm{~m}^{2}\right)(10,13,15-17,26)$, which provides the support for the current K/DOQI guidelines in CKD and KDIGO in transplant $(8,30)$. Special attention, however, must be paid toward its specific limitations in various clinical settings, such as children with reduced muscle mass, hyperfiltration (GFR $>120 \mathrm{ml} / \mathrm{min} / 1.73 \mathrm{~m}^{2}$ ), and older than 15 years where a measured GFR or addition of other GFR markers may provide a better assessment. Further studies are required to continuously improve the precision and accuracy of eGFR measurements so that it can be done simply and cost effectively.

\section{SUPPLEMENTARY MATERIAL}

Supplementary material is linked to the online version of the paper at http://www.nature.com/pr

\section{ACKNOWLEDGMENTS}

We thank the participants, families, and healthcare providers in the Renal Transplant Program at the Hospital for Sick Children. We thank Christopher Battiston for his support and retrieval of electronic data, and Michelle Estrella for her analytical advice.

Disclosure: The authors declare no conflict of interest.

\section{REFERENCES}

1. Goolsby MJ. National Kidney Foundation Guidelines for chronic kidney disease: evaluation, classification, and stratification. J Am Acad Nurse Pract 2002;14:238-42.

2. Fellstrom B, Jardine AG, Soveri I, et al. Renal dysfunction as a risk factor for mortality and cardiovascular disease in renal transplantation: experience from the Assessment of Lescol in Renal Transplantation trial. Transplantation 2005;79:1160-3.

3. Briggs JD. Causes of death after renal transplantation. Nephrol Dial Transplant 2001;16:1545-9.

4. First MR. Renal function as a predictor of long-term graft survival in renal transplant patients. Nephrol Dial Transplant 2003;18 (Suppl 1): i3-6.

5. Perrone RD, Steinman TI, Beck GJ, et al. Utility of radioisotopic filtration markers in chronic renal insufficiency: simultaneous comparison of 125I-iothalamate, 169Yb-DTPA, 99mTc-DTPA, and inulin. The Modification of Diet in Renal Disease Study. Am J Kidney Dis 1990;16: 224-35.

6. The 2007 Recommendations of the International Commission on Radiological Protection. ICRP publication 103. Ann ICRP 2007; 37:1-332.

7. Bokenkamp A, Domanetzki M, Zinck R, Schumann G, Byrd D, Brodehl J. Cystatin C-a new marker of glomerular filtration rate in children independent of age and height. Pediatrics 1998;101:875-81.
8. Kidney Disease: Improving Global Outcomes Transplant Work G. KDIGO clinical practice guideline for the care of kidney transplant recipients. Am J Transplant 2009;9 (Suppl 3): S1-155.

9. Schwartz GJ, Haycock GB, Edelmann CM Jr, Spitzer A. A simple estimate of glomerular filtration rate in children derived from body length and plasma creatinine. Pediatrics 1976;58:259-63.

10. Schwartz GJ, Munoz A, Schneider MF, et al. New equations to estimate GFR in children with CKD. J Am Soc Nephrol 2009;20:629-37.

11. Zappitelli M, Joseph L, Gupta IR, Bell L, Paradis G. Validation of child serum creatinine-based prediction equations for glomerular filtration rate. Pediatr Nephrol 2007;22:272-81.

12. Staples A, LeBlond R, Watkins S, Wong C, Brandt J. Validation of the revised Schwartz estimating equation in a predominantly non-CKD population. Pediatr Nephrol 2010;25:2321-6.

13. De Souza VC, Rabilloud M, Cochat P, et al. Schwartz formula: is one k-coefficient adequate for all children? PLoS ONE 2012;7:e53439.

14. Tsampalieros A, Lepage N, Feber J. Intraindividual variability of the modified Schwartz and novel CKiD GFR equations in pediatric renal transplant patients. Pediatr Transplant 2011;15:760-5.

15. Papez KE, Barletta GM, Hsieh S, Joseph M, Morgenstern BZ. Iothalamate versus estimated GFR in a Hispanic-dominant pediatric renal transplant population. Pediatr Nephrol 2013;28:2369-76.

16. Siddique K, Leonard D, Borders L, Seikaly MG. Validation of the CKiD formulae to estimate GFR in children post renal transplant. Pediatr Nephrol 2014;29:445-51.

17. de Souza V, Cochat P, Rabilloud M, et al. Accuracy of different equations in estimating GFR in pediatric kidney transplant recipients. Clin J Am Soc Nephrol 2015;10:463-70.

18. Zappitelli M, Zhang X, Foster BJ. Estimating glomerular filtration rate in children at serial follow-up when height is unknown. Clin J Am Soc Nephrol 2010;5:1763-9.

19. Pottel H, Hoste L, Martens F. A simple height-independent equation for estimating glomerular filtration rate in children. Pediatr Nephrol 2012;27: 973-9.

20. Ylinen EA, Ala-Houhala M, Harmoinen AP, Knip M. Cystatin C as a marker for glomerular filtration rate in pediatric patients. Pediatr Nephrol 1999;13:506-9.

21. Stickle D, Cole B, Hock K, Hruska KA, Scott MG. Correlation of plasma concentrations of cystatin $\mathrm{C}$ and creatinine to inulin clearance in a pediatric population. Clin Chem 1998;44:1334-8.

22. Schwartz GJ, Schneider MF, Maier PS, et al. Improved equations estimating GFR in children with chronic kidney disease using an immunonephelometric determination of cystatin C. Kidney Int 2012;82:445-53.

23. Inker LA, Schmid CH, Tighiouart $\mathrm{H}$, et al. Estimating glomerular filtration rate from serum creatinine and cystatin C. N Engl J Med 2012;367:20-9.

24. Filler G, Lepage N. Should the Schwartz formula for estimation of GFR be replaced by cystatin C formula? Pediatr Nephrol 2003;18:981-5.

25. Dworkin LD. Serum cystatin $\mathrm{C}$ as a marker of glomerular filtration rate. Curr Opin Nephrol Hypertens 2001;10:551-3.

26. Westland R, Abraham Y, Bokenkamp A, Stoffel-Wagner B, Schreuder MF, van Wijk JA. Precision of estimating equations for GFR in children with a solitary functioning kidney: the KIMONO study. Clin J Am Soc Nephrol 2013;8:764-72.

27. Blufpand HN, Westland R, van Wijk JA, Roelandse-Koop EA, Kaspers GJ, Bokenkamp A. Height-independent estimation of glomerular filtration rate in children: an alternative to the Schwartz equation. J Pediatr 2013;163:1722-7.

28. Bland JM, Altman DG. Agreement between methods of measurement with multiple observations per individual. J Biopharm Stat 2007;17:571-82.

29. Bland JM, Altman DG. Measuring agreement in method comparison studies. Stat Methods Med Res 1999;8:135-60.

30. National Kidney F. K/DOQI clinical practice guidelines for chronic kidney disease: evaluation, classification, and stratification. Am J Kidney Dis 2002;39:S1-266.

31. Bland JM, Altman DG. Statistical methods for assessing agreement between two methods of clinical measurement. Lancet 1986;1:307-10. 


\section{Articles | Alkandari et al.}

32. Chavers BM, Rheault MN, Foley RN. Kidney function reference values in US adolescents: National Health And Nutrition Examination Survey 1999-2008. Clin J Am Soc Nephrol 2011;6:1956-62.

33. Groesbeck D, Kottgen A, Parekh R, et al. Age, gender, and race effects on cystatin C levels in US adolescents. Clin J Am Soc Nephrol 2008;3:1777-85.
34. Schwartz GJ, Brion LP, Spitzer A. The use of plasma creatinine concentration for estimating glomerular filtration rate in infants, children, and adolescents. Pediatr Clin North Am 1987;34:571-90.

35. Filler G, Sharma AP. How to monitor renal function in pediatric solid organ transplant recipients. Pediatr Transplant 2008;12:393-401. 